\title{
Estado nutricional e prevalência de enteroparasitoses em crianças matriculadas em creche
}

\author{
Nutritional status and enteroparasitosis prevalence among children enrolled in a day care center
}

\begin{abstract}
Terezinha Soares Biscegli ${ }^{1}$, João Romera ${ }^{2}$, André Binotti Candido ${ }^{3}$, Jaine Maria dos Santos ${ }^{4}$, Ellen Cristina A. Candido ${ }^{3}$, André Luiz Binotto ${ }^{3}$
\end{abstract}

\section{RESUMO}

Objetivo: Avaliar o estado nutricional e descrever a prevalência de enteroparasitoses em crianças de uma creche.

Métodos: Estudo transversal com 133 crianças (sete a 78 meses de idade) matriculadas na Creche "Sinharinha Neto", Catanduva (SP). Avaliou-se o estado nutricional pelas curvas da OMS/2006 e NCHS/2000 (menores e maiores de cinco anos, respectivamente) e investigou-se enteroparasitoses por meio de exames parasitológicos de fezes. O escore Z dos índices peso/altura foi aplicado para classificar desnutrição aguda $(Z<-2)$ e obesidade $(Z>2)$ e o da altura/idade para desnutrição pregressa e crônica $(Z<-2)$. Para análise estatística das variáveis, utilizou-se o teste $Z$ para duas proporções, sendo significante $p \leq 0,05$.

Resultados: Observou-se $0,8 \%$ de desnutrição aguda, $1,5 \%$ de desnutrição pregressa e $6 \%$ de obesidade. A prevalência de distúrbios nutricionais foi significativamente maior no gênero masculino. A prevalência de enteroparasitoses foi de 29,2\%. Os parasitas encontrados foram Giardia lamblia $(73,6 \%)$, Entamoeba coli e leveduras (10,5\% cada) e Endolimax nana (7,9\%). A faixa etária mais acometida foi de 25 a 60 meses (60,5\%). A prevalência de Entamoeba coli e leveduras foi significativamente maior em crianças desnutridas em comparação às eutróficas.

Conclusões: A transição nutricional é comprovada nas crianças da creche "Sinharinha Neto". A melhora na qualidade de vida contribuiu para o desaparecimento de algumas parasitoses, mas não afetou de forma efetiva a ocorrência de giardíase. Seria benéfico adotar medidas preventivas contra a obesidade infantil e as protozooses, tanto na creche como nos domicílios.

Instituição: Faculdades Integradas Padre Albino (FIPA), Catanduva, SP, Brasil ${ }^{1}$ Pediatra; Doutora em Medicina pela Faculdade de Medicina de Ribeirão Preto da Universidade de São Paulo; Professora e Coordenadora da Área Materno-Infantil do Curso de Medicina das FIPA, Catanduva, SP, Brasil ${ }^{2}$ Pediatra; Professor da Disciplina de Puericultura e Clínica Pediátrica do Curso de Medicina das FIPA, Catanduva, SP, Brasil

${ }^{3}$ Médico, graduado pelo Curso de Medicina das FIPA, Catanduva, SP, Brasil

${ }^{4}$ Aluna do Curso de Medicina das FIPA, Catanduva, SP, Brasil
Palavras-chave: criança; creches; estado nutricional; parasitoses.

\section{ABSTRACT}

Objective: To evaluate the nutritional status and the prevalence of intestinal parasitic diseases among children enrolled in a day care center.

Methods: This cross-sectional study comprised 133 children, aged seven to 78 months, assisted in a nonprofit day care center in Catanduva, SP, Brazil. The nutritional status was classified according to WHO/2006 and NCHS/2000 curves (younger and older than five years, respectively). Faecal samples were collected for parasite examination. Z scores of weight/height were used to classify acute malnutrition $(Z<-2)$ and obesity $(Z>2)$, and height/age to classify past and chronic malnutrition $\left(Z_{<-2}\right)$. Statistical analyses were performed by $Z$ test for proportions, beeing significant $\mathrm{p} \leq 0.05$.

Results: Among the evaluated children, $0.8 \%$ presented acute malnutrition, $1.5 \%$ past malnutrition and $6 \%$ obesity. Nutritional disorders were more prevalent among boys. The prevalence of intestinal parasites was $29.2 \%$. The parasites identified were Giardia lamblia (73.6\%), Entamoeba coli and yeasts (10.5\% each) and Endolimax nana (7.9\%). Children aged 25 to 60 months presented more parasites (60.5\%). Entamoeba coli and yeasts were more prevalent among malnourished children in comparison to eutrophic children.

Conclusions: Nutritional transition was observed in this study. Improvement in life conditions contributed to the disappearance of some parasites, but not of giardiasis. It is

Endereço para correspondência:

Terezinha Soares Biscegli

Rua Fortaleza, 95 - Jardim Ferreira

CEP 15840-000 - Itajobi/SP

E-mail: terezinhabiscegli@yahoo.com.br

Recebido em: 7/11/08

Aprovado em: 1/3/09 
important to adopt effective preventive measures against childhood obesity and protozoan infestations in the day care center as much as in children's homes.

Key-words: child; child day care centers; nutritional status; parasitic diseases.

\section{Introdução}

As enteroparasitoses são consideradas um problema de saúde pública no Brasil e, habitualmente, estão associadas ao baixo nível socioeconômico da população. Representam fator importante na etiologia das anemias carenciais e da desnutrição proteico-calórica, pois um estado nutricional adequado depende não só da ingestão dos alimentos, mas também de sua utilização biológica eficiente, que pode estar comprometida em casos de infestação por enteroparasitas $^{(1,2)}$.

Sabe-se que a desnutrição em fases precoces da vida promove redução da capacidade de realizar trabalho, maior vulnerabilidade às infecções, menor capacidade cognitiva, diminuição na biotransformação metabólica e má-absorção intestinal de nutrientes ${ }^{(2)}$. É fato conhecido também que as enteroparasitoses são mais frequentes em regiões menos desenvolvidas ${ }^{(3)}$. Dados da Pesquisa Nacional de Demografia e Saúde (PNDS) ${ }^{(4)}$ mostraram que a prevalência da desnutrição em crianças menores de cinco anos, aferida pela proporção de déficit de crescimento, foi $7 \%$ em 2006. A maior prevalência ocorreu na região Norte $(15 \%)$ e houve pouca variação nas demais regiões (6 a 8\%). Casos agudos de desnutrição (déficit de peso em relação à altura) foram encontrados em apenas 1,5\% das crianças menores de cinco anos, não ultrapassando $2 \%$ em qualquer região ou estrato social da população, o que indica que as formas agudas de deficiência energética estão praticamente sob controle em todo o país. Por outro lado, situações de excesso de peso em relação à altura foram encontradas em $7 \%$ das crianças brasileiras menores de cinco anos (variando de $6 \%$ na região Norte a $9 \%$ na Sul), alertando para o problema da obesidade infantil ${ }^{(4)}$.

Apesar da aparente melhora do quadro nutricional das crianças do Brasil, estima-se que milhões de indivíduos estejam infestados por enteroparasitas. No Estado de São Paulo, observou-se diminuição na prevalência de infestação por enteroparasitas nos últimos 30 anos. No entanto, os índices ainda são bastante significativos ${ }^{(5)}$. Estudo desenvolvido em meados de 1980 por pesquisadores do município de São Paulo com crianças de idade inferior a cinco anos mostrou que $31 \%$ delas apresentavam uma ou mais espécies de parasitas intestinais (mais frequentemente áscaris, giárdia e tricocéfalos), todos com prevalência acima de $10 \%$. O poliparasitismo intestinal atingiu $13 \%$ da amostra, sendo mais elevado nas crianças de nível socioeconômico mais baixo. O predomínio da ascaridíase, giardíase e tricocefalíase é referido na maioria dos países do Terceiro Mundo ${ }^{(6)}$. No município de Catanduva, em estudo realizado em 1991, a frequência de enteroparasitoses foi de $51 \%$, sendo os mais prevalentes Giardia lamblia (26\%) e Entamoeba coli $(23 \%)^{(7)}$. Um estudo mais recente no mesmo município, com crianças de escola pública, demonstrou que a incidência de parasitoses diminuiu em comparação ao estudo anterior (13\%), e que o parasita predominante continuou sendo a Giardia lamblia $(44 \%)^{(8)}$.

Existe uma falta de dados disponíveis sobre morbidade e mortalidade decorrentes das parasitoses intestinais, geralmente não notificadas ou subnotificadas pelos serviços de saúde. Por serem considerados "problemas menores", os profissionais da área acabam demonstrando pouco interesse, além de uma atitude tolerante ${ }^{(6)}$.

A creche, nos dias atuais, é uma realidade na vida das crianças e, consequentemente, do pediatra. É o local onde muitas crianças passam a maior parte de sua infância e, com isso, fica claro o papel importante que essa instituição tem no desenvolvimento integral da criança em seus aspectos físico, psicológico, intelectual e social ${ }^{(9)}$. Uma creche adequada é capaz de favorecer a prevenção e o diagnóstico precoce de alguns problemas de saúde, além de estimular as crianças em suas diversas etapas do desenvolvimento. No entanto, as crianças de creche estão mais sujeitas a infecções por causa do grande contato com outras crianças e adultos e, frequentemente, apresentam mais problemas gastrintestinais, de pele, doenças infecto-contagiosas, respiratórias, incluindo as otites ${ }^{(10)}$.

Considerando-se os efeitos prejudiciais dos distúrbios nutricionais e das enteroparasitoses sobre o crescimento e o desenvolvimento infantil e os benefícios advindos de sua prevenção e tratamento precoces, o objetivo do presente estudo é avaliar o estado nutricional e descrever a prevalência de enteroparasitoses nas crianças que frequentam a creche "Sinharinha Neto", em Catanduva (SP).

\section{Métodos}

Estudo transversal realizado no período de setembro a novembro de 2007 com 133 crianças matriculadas na 
creche "Sinharinha Neto", na cidade de Catanduva, São Paulo, todas classificadas como de "baixa renda" de acordo com os critérios estabelecidos pela própria creche, que leva em consideração vários fatores como renda familiar, tipo de moradia, composição familiar e necessidade da mãe trabalhar fora de casa.

Das 144 crianças que frequentavam a creche durante o período do estudo, 11 foram excluídas porque os pais não permitiram a sua participação na pesquisa. Realizou-se a avaliação nutricional nas 133 crianças participantes (de sete a 78 meses de idade) e a investigação de parasitoses intestinais em 130 delas (98\%), pois em três crianças não foi possível coletar fezes nos dias programados. Todas as crianças apresentavam-se assintomáticas nos dias de investigação.

A ficha de matrícula da criança foi utilizada para determinar a idade cronológica (em meses) e o gênero. A antropometria (peso e estatura) obedeceu às técnicas preconizadas pela Organização Mundial de Saúde (OMS) (11) e foi realizada exclusivamente pelos autores, especialmente treinados para desempenhar tal tarefa. A medição do comprimento da criança de zero a 24 meses foi feita em decúbito dorsal (antropômetro horizontal) e, para as maiores de dois anos, aferiu-se a altura em posição ortostática (antropômetro vertical). A aferição do peso foi realizada com balança pediátrica (precisão de $10 \mathrm{~g}$ ) e balança plataforma (precisão de $100 \mathrm{~g}$ ), para crianças de até $15 \mathrm{~kg}$ e acima de $15 \mathrm{~kg}$ respectivamente.

Para a avaliação nutricional, utilizaram-se as curvas da Organização Mundial de Saúde (OMS) ${ }^{(12)}$ e do National Center for Health Statistics (NCHS) ${ }^{(13)}$ (respectivamente para crianças menores e maiores de cinco anos). Foram consideradas as relações entre peso/altura (P/A) e altura/idade (A/I), sendo o escore $Z \leq-2$ e $Z \geq 2$, respectivamente, utilizados para definir desnutrição e obesidade. Foram consideradas eutróficas as crianças que apresentaram relação $\mathrm{P} / \mathrm{A}$ e $\mathrm{A} / \mathrm{I}$ normais; com desnutrição aguda as que apresentaram $\mathrm{P} / \mathrm{A} \leq-2$ e $\mathrm{A} / \mathrm{I}$ normal; e com desnutrição pregressa aquelas que apresentaram $\mathrm{P} / \mathrm{A}$ normal e $\mathrm{A} / \mathrm{I} \leq-2$. Foram classificadas como obesas as crianças que apresentaram $\mathrm{P} / \mathrm{A} \geq 2$.

A investigação de parasitoses foi realizada por exames parasitológicos de fezes. As fezes foram colhidas em dias alternados pelos responsáveis das crianças ou por um funcionário da creche treinado para desempenhar essa tarefa. Três amostras de fezes foram colhidas de 50 participantes, duas amostras de 73 e uma única amostra de 10 crianças. O material foi acondicionado em frasco apropriado fornecido pelo laboratório e imediatamente encaminhado para análise. As técnicas de análise das fezes foram: exame direto a fresco, método de Hoffman e método de Baermann ${ }^{(14)}$.

O presente projeto de pesquisa foi aprovado pelo Comitê de Ética em Pesquisa da Faculdade de Medicina de Catanduva e foi solicitada a assinatura do termo de consentimento livre e esclarecido pelos responsáveis legais das crianças. Os resultados foram expressos em porcentagem. Para a análise estatística das variáveis de interesse, utilizou-se o teste $\mathbf{Z}$ para duas proporções. Considerou-se significante $p \leq 0,05$.

\section{Resultados}

Das 133 crianças estudadas, 69 (52\%) eram do gênero masculino e 64 (48\%) do feminino. A idade variou de sete a 78 meses. A faixa etária predominante foi de 25 a 60 meses com 76 crianças ( $57 \%$ ), seguida das faixas etárias de maiores de cinco anos com 33 crianças $(25 \%)$ e de menores de dois anos com 24 crianças (18\%).

A avaliação do estado nutricional está apresentada na Tabela 1. Os distúrbios nutricionais encontrados, de acordo com a faixa etária e o gênero, são mostrados na Tabela 2. Não foi detectado nenhum caso de desnutrição crônica. A prevalência de distúrbios nutricionais foi maior no gênero masculino comparada ao feminino $(p<0,03)$.

A prevalência de enteroparasitoses foi de 29\% (38 crianças com resultado positivo para um ou mais parasitas). A Giardia lamblia foi o parasita mais encontrado (74\%). Em segundo lugar ficaram Entamoeba coli e leveduras (10\% cada) e o menos frequente foi Endolimax nana (8\%). Os resultados dos exames de fezes estão demonstrados no Gráfico 1. Das crianças parasitadas, $22(58 \%)$ eram do gênero masculino e $16(42 \%)$ do feminino. A faixa etária mais acometida foi a dos 25 aos 60 meses ( 23 crianças $-60 \%$ ), seguida dos menores de dois anos (12 crianças - 32\%), e finalmente das maiores de cinco anos, com apenas três crianças (8\%).

Tabela 1 - Distribuição das crianças matriculadas na Creche "Sinharinha Neto", em Catanduva (SP), no ano de 2007, de acordo com o estado nutricional

\begin{tabular}{lrr}
\hline Estado nutricional & $\mathbf{n}$ & $\%$ \\
\hline Eutrofia & 122 & 91,7 \\
Desnutrição aguda & 1 & 0,8 \\
Desnutrição pregressa & 2 & 1,5 \\
Obesidade & 8 & 6,0 \\
\hline
\end{tabular}


Tabela 2 - Distribuição dos distúrbios nutricionais encontrados em crianças matriculadas na Creche "Sinharinha Neto", em Catanduva (SP), no ano de 2007, de acordo com a faixa etária e o gênero

\begin{tabular}{lcccccc}
\hline & \multicolumn{2}{c}{$\mathbf{0}$ a 24 meses } & \multicolumn{2}{c}{$\mathbf{2 5}$ a $\mathbf{6 0}$ meses } & \multicolumn{2}{c}{ Mais de $\mathbf{6 0}$ meses } \\
\hline Gênero & $\mathrm{M}$ & $\mathrm{F}$ & $\mathrm{M}$ & $\mathrm{F}$ & $\mathrm{M}$ & $\mathrm{F}$ \\
Desnutrição aguda & 0 & 0 & $1(100 \%)$ & 0 & 0 & 0 \\
Desnutrição pregressa & $1(50 \%)$ & 0 & $1(50 \%)$ & 0 & 0 & 0 \\
Obesidade & 0 & 0 & $3(37 \%)$ & $2(25 \%)$ & $2(25 \%)$ & $1(12 \%)$ \\
\hline
\end{tabular}

Tabela 3 - Distribuição dos enteroparasitas encontrados em crianças matriculadas na Creche "Sinharinha Neto", em Catanduva (SP), no ano de 2007, de acordo com o estado nutricional

\begin{tabular}{lcccc}
\hline & Giardia lamblia & Entamoeba coli & Endolimax nana & Leveduras \\
\hline Eutrofia & $25(20 \%)$ & $2(2 \%)$ & $3(2 \%)$ & $2(2 \%)$ \\
Desnutrição aguda & 0 & $1(100 \%)$ & 0 & 0 \\
Desnutrição pregressa* & $1(50 \%)$ & $1(50 \%)$ & 0 & $1(50 \%)$ \\
Obesidade & $2(25 \%)$ & 0 & 0 & $1(12 \%)$ \\
\hline
\end{tabular}

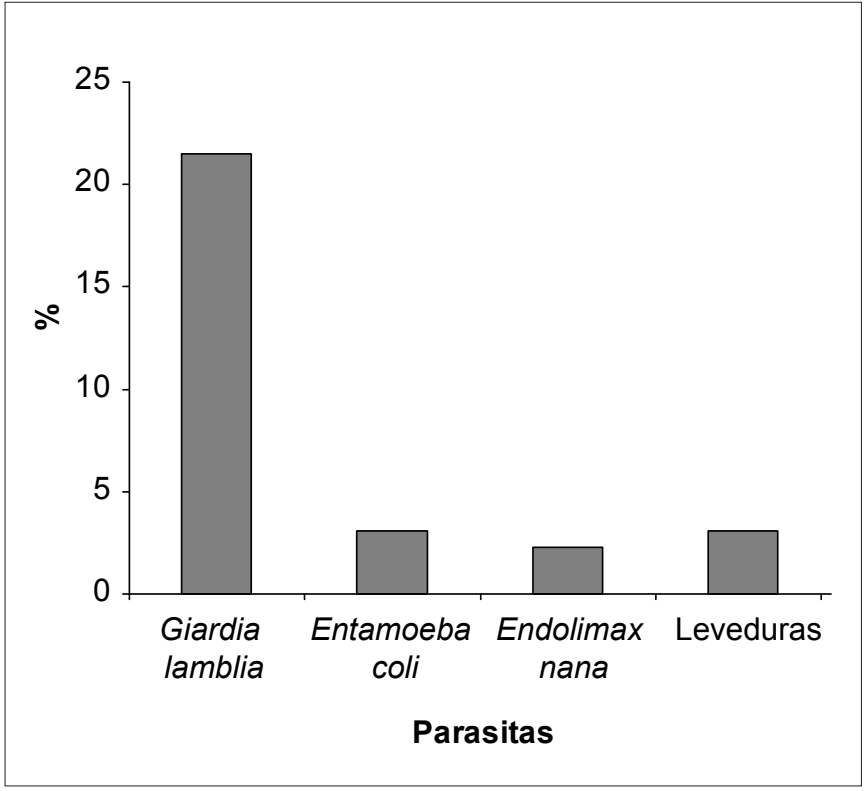

Gráfico 1 - Prevalência de enteroparasitoses em crianças de sete a 78 meses de idade da creche "Sinharinha Neto", em Catanduva (SP), em 2007.

A distribuição dos parasitas de acordo com o estado nutricional está registrada na Tabela 3. Observou-se maior prevalência de Giardia lamblia nas crianças portadoras de desnutrição pregressa quando comparadas às eutróficas, embora esta diferença não tenha sido significante $(p=0,31)$. Já a prevalência de Entamoeba coli e leveduras foi maior nas crianças com desnutrição em comparação às eutróficas $(p<0,001)$.

\section{Discussão}

A análise dos dados antropométricos das crianças frequentadoras da creche "Sinharinha Neto" revelou que a maioria delas apresentou-se eutrófica e que a obesidade prevaleceu sobre a desnutrição. Resultados semelhantes foram observados em outro estudo que evidenciou um aumento da obesidade em todas as camadas da população, mas, principalmente, em famílias de menor poder aquisitivo ${ }^{(15)}$. Nos últimos 30 anos, observa-se mudança nos padrões nutricionais da população brasileira (transição nutricional), com diminuição evidente de desnutridos e aumento da frequência de indivíduos com sobrepeso ou obesidade, principalmente devido aos hábitos alimentares inadequados ${ }^{(16)}$. Este fato já é motivo de preocupação em nível de Saúde Pública, pois a presença de obesidade leva a um aumento das taxas de morbidade e de doenças crônicas como diabetes, problemas cardiovasculares, ortopédicos e distúrbios psicológicos e sociais ${ }^{(17)}$.

Dados recentes de uma pesquisa desenvolvida na cidade de Catanduva com crianças de creche também demonstraram predomínio de obesidade sobre desnutrição, na proporção de $4: 1^{(18)}$. Outro estudo com crianças de creche da cidade de Catanduva ${ }^{(19)}$ também evidenciou maior número de obesos $(15,9 \%)$ comparado ao de desnutridos $(11,5 \%)$, embora a prevalência de distúrbios nutricionais tenha sido consideravelmente maior. Esta diferença pode ser devido ao fato de a creche em questão se localizar em bairro de periferia, abrangendo crianças de situação econômica menos favorecida. Estudo atual, publicado em 
2008 e realizado com crianças de creche de um município do Rio de Janeiro, também mostrou reflexos da transição nutricional ${ }^{(20)}$. Resultados diferentes foram obtidos em um estudo de 2005, desenvolvido em creches beneficentes do município de São Paulo, que demonstrou prevalência similar de desnutrição $(7,4 \%)$ e obesidade $(6,3 \%)^{(21)}$. Em nossa pesquisa, a prevalência de desnutridos $(0,8 \%$ com desnutrição aguda e 1,5\% com desnutrição pregressa) foi baixa em comparação à média populacional dos países em desenvolvimento, inclusive o $\mathrm{Brasil}^{(4)}$. Comparando-se os resultados do atual com os de um estudo anterior ${ }^{(17)}$, desenvolvido com crianças da mesma creche, observou-se que a prevalência de desnutrição diminuiu, enquanto a de obesidade permaneceu semelhante.

Crianças que frequentam creche recebem praticamente $70 \%$ de suas necessidades nutricionais por meio de alimentação balanceada, durante praticamente dez horas por dia. Em decorrência disto, deveriam estar mais protegidas contra distúrbios nutricionais em relação à população geral ${ }^{(22)}$. A alimentação das crianças estudadas foi elaborada por um nutricionista (dieta geral, adequada para a idade), oferecida em horários controlados. Dessa forma, acredita-se que os casos de obesidade observados (6\%), embora compatíveis com a média nacional, devem alertar que os fatores desencadeadores possam estar relacionados ao ambiente familiar (após a saída da creche, nos finais de semana, feriados e férias), já que, na creche, as transgressões alimentares são pouco prováveis. Outro fator que pode estar contribuindo para a obesidade é a falta de atividades esportivas aeróbicas na creche.

De acordo com os resultados obtidos, verifica-se que a prevalência de desnutrição está em franca redução, mas que a prevalência de obesidade vem se tornando um fato preocupante na população de baixa renda ${ }^{(16)}$. As crianças que apresentaram distúrbio nutricional foram encaminhadas a profissionais especializados para avaliação mais acurada e intervenções pertinentes.

A análise dos exames de fezes das crianças frequentadoras da creche "Sinharinha Neto" detectou prevalência de enteroparasitoses em $29 \%$ dos casos. Há a possibilidade de que esta prevalência esteja subestimada, pois a coleta de três amostras de fezes, que melhoraria a positividade do exame protoparasitológico, só foi realizada em $38 \%$ dos participantes. Uma pesquisa realizada neste mesmo município, no ano de 2004, com crianças de escola pública, encontrou prevalência consideravelmente menor $(13 \%)^{(8)}$.

O protozoário predominante em ambos os estudos foi a Giardia lamblia (74 e 44\%, respectivamente). Frequências semelhantes foram encontradas em outros estudos brasileiros que também apontaram a giardíase como a principal enteroparasitose brasileira ${ }^{(23,24)}$. Crianças frequentadoras de creches, assim como as pessoas que lá trabalham, apresentam altas taxas (17 a 47\%) de infecção por Giardia lamblia, manifestada de forma clínica ou subclínica ${ }^{(25)}$. Sabe-se que quando uma criança apresenta giardíase, há 5 a $25 \%$ de chance de um ou mais membros da família estarem contaminados ${ }^{(25,26)}$. A giardíase tem sido alvo de pesquisas sobre síndromes diarreicas em crianças de creche. Também bastante discutida é a questão da 'proteção' ou 'exposição' que essas unidades de cuidados propiciam às crianças ${ }^{(27)}$. A profilaxia da giardíase pode ser feita por meio de recomendações simples, tais como consumo de água fervida ou filtrada, higiene dietética adequada, orientações de higiene pessoal, destino adequado aos dejetos e, finalmente, o tratamento dos indivíduos doentes e, em determinadas situações, dos supostamente infestados ${ }^{(6)}$.

Comparada a estudos descritos anteriormente ${ }^{(8,23-27)}$, chama a atenção, na presente pesquisa, o grande número de crianças contaminadas por Giardia lamblia e a negatividade das amostras para outros parasitas, tais como helmintos. No caso da giardíase, isso pode ser devido ao fato de os cistos desses protozoários serem resistentes ao tratamento da água e também ao hábito de ingerir água não filtrada e não fervida. Quanto à ausência de helmintíase, sugere-se uma relação com o uso sistemático de automedicação, tão comum em nosso meio, além da limitação apresentada pelo estudo no que concerne à coleta de três amostras de fezes. Apesar de a prevalência de giardíase ter sido elevada, os dados mostram que não houve prejuízo do estado nutricional das crianças estudadas (apenas 2,3\% de desnutrição). Os casos de giardíase foram tratados com metronidazol, na dose de $20 \mathrm{mg} / \mathrm{kg} /$ dia por um período de sete dias ${ }^{(6)}$.

Das crianças pesquisadas contaminadas com enteroparasitas, a minoria apresentou resultado positivo para protozoários não enteropatogênicos, tais como Entamoeba coli $(10,5 \%)$ e Endolimax nana $(7,9 \%)$. Um estudo realizado na Colômbia, com 423 pacientes, demonstrou prevalência consideravelmente maior (Entamoeba coli, 27,9\% e Endolimax nana, 20,3\%) ${ }^{(28)}$. Entretanto, vale lembrar que as populações estudadas eram diferentes em vários aspectos. Outra investigação, desenvolvida com crianças de uma escola pública da cidade de Catanduva, demonstrou que a Entamoeba coli $(31,25 \%)$ e o Endolimax nana $(15,62 \%)$ estavam entre os parasitas mais encontrados ${ }^{(8)}$. Quadros $e t$ al, ao analisarem crianças de dois a seis anos de centros de educação infantil do Rio Grande do Sul, demonstraram que 70,5\% das crianças 
tiveram pelo menos uma infecção parasitária, embora a prevalência de Entamoeba coli (4,5\%) e Endolimax nana (0,5\%) tivessem sido consideravelmente menor do que a observada pelo presente estudo ${ }^{(29)}$.

Outro aspecto a ser considerado foi a detecção de leveduras nas fezes de quatro $(10,5 \%)$ das 38 crianças cujo exame de fezes foi positivo. Vale lembrar que todas as crianças se apresentavam assintomáticas no momento dos exames. Sabe-se que as leveduras estão amplamente distribuídas no ambiente, sendo também habitantes normais do corpo humano. São consideradas patógenos oportunistas, causadores de infecções que variam desde superficiais até profundas e fatais ${ }^{(30)}$. Essas infecções não têm limitação geográfica porque ocorrem principalmente em pacientes com predisposição a elas $^{(31,32)}$. Em um estudo desenvolvido com indivíduos aparentemente saudáveis e com pessoas que mostravam sintomas de infecção fúngica, detectou-se a presença de leveduras em 77,5\% das amostras fecais, sendo a Candida albicans a espécie mais prevalente ${ }^{(30)}$. Essa divergência de resultados com relação à presente pesquisa pode ser explicada pela inclusão de indivíduos sintomáticos no último estudo ${ }^{(30)}$. Forbes et al, em pesquisa realizada com crianças hospitalizadas, demonstraram que a Candida spp não causa diarreia em crianças eutróficas, mas desempenha papel importante em desnutridos com diarreia crônica ${ }^{(33)}$.

\section{Referências bibliográficas}

1. WHO (World Health Organization), United Nations Protein Advisory Group. Anthropometry in nutritional surveillance: an overview. Bull World Health Organ 1976;6:2-11.

2. Ferreira HS. Desnutrição, magnitude, significado social e possibilidade de prevenção. Maceió (AL): Edufal; 2000.

3. Sigulem DM, Tudisco ES, Paiva ER, Guerra CC. Anemia nutricional e parasitose intestinal em menores de 5 anos. Rev Paul Med 1985;103: 308-12.

4. Brasil. Ministério da Saúde. Pesquisa Nacional de Demografia e Saúde da Criança e da Mulher (PNDS-2006) [homepage in the Internet]. Saúde Nutricional. Saúde e estado nutricional de crianças menores de cinco anos [cited $2009 \mathrm{Jul}$ 23]. Available from: http://bvsms.saude.gov.br/bvs/pnds/ saude_nutricional.php

5. Chieffi PP, Gryschek RC, Amato Neto V. Parasitoses intestinais: diagnóstico e tratamento. São Paulo: Lemos Editorial; 2001.

6. Bresolin AM, Zuccolotto SM. Parasitoses intestinais. In: Marcondes E, Vaz FA, Okay Y, Ramos JL, editores. Pediatria básica: pediatria clínica geral. $9^{a}$ ed. São Paulo: Sarvier; 2003. p. 264-79.

7. Pinto PLS, Cerqueira RL, Ribeiro EA, Matheus RCS, Cury JC. Aspectos epidemiológicos das parasitoses intestinais em crianças menores de 14 anos residentes no município de Catanduva (SP). Klinikos 1991;6:20-2.

8. Faleiros JM, Gallo G, Silva MM, Raful R, Nasorri AR, Pipino LF et al. Ocorrência de enteroparasitoses em alunos da escola pública de ensino
Pode-se concluir que a transição nutricional está ocorrendo nas crianças que frequentam a creche "Sinharinha Neto" e que a melhora na qualidade de vida, incluindo saneamento básico e educação sanitária, contribui para o desaparecimento de algumas enteroparasitoses. Entretanto, deve-se alertar para a necessidade da adoção de medidas preventivas contra a obesidade infantil e especificamente contra a giardíase, tanto na creche como no domicílio. A prática de atividades aeróbicas diárias poderia ser implementada pela creche. Também seria interessante fornecer orientação dietética às mães e incentivar a prática de esportes nos finais de semana. $\mathrm{O}$ acompanhamento por pediatra e nutricionista na própria creche seria benéfico para detectar e intervir precocemente nos casos de distúrbios. A investigação de giardíase nos familiares das crianças e no pessoal de apoio da creche poderia colaborar para reduzir esta protozoose. Dessa maneira, a promoção da saúde infantil seria realizada em conjunto: pela creche e pela família.

\section{Agradecimentos}

À Sra. Maria Helena Denadai, coordenadora da creche "Sinharinha Neto", à Srta. Margarida Marioti, recepcionista do Hospital Emílio Carlos (in memoriam) pelo auxílio na parte operacional, e aos professores, funcionários, pais e crianças pela participação e interesse.

fundamental do município de Catanduva (São Paulo, Brasil). Rev Inst Adolfo Lutz 2004;63:243-7.

9. Brasil. Ministério da Educação [homepage on the Internet]. Diretrizes e bases da educação nacional: lei nº 9.394, 1996 [cited 2009 Jul 23]. Available from: http://www.planalto.gov.br/ccivil_03/Leis/L9394.htm

10. Alderete JM, Douek PC. A criança e a creche. In: Marcondes E, Vaz FA, Okay Y, Ramos JL, editores. Pediatria básica: pediatria geral e neonatal. $9^{\mathrm{a}} \mathrm{ed}$. São Paulo: Sarvier; 2002. p. 610-3.

11. WHO. Physical status: the use and interpretation of anthropometry. Report of a WHO expert committee. Geneva: WHO; 1995.

12. de Onis M, Garza C, Victora CG, Onyango AW, Frongillo EA, Martines J. The WHO Multicentre Growth Reference Study: planning, study design, and methodology. Food Nutr Bull 2004;25(Suppl 1):S15-26.

13. NCHS [homepage on the Internet]. 2000 CDC growth charts: United States [cited 2009 Jul 23]. Available from: http://www.cdc.gov/growthcharts/

14. De Carli GA. Parasitologia clínica: seleção de métodos e técnicas de laboratório para o diagnóstico das parasitoses humanas. São Paulo: Atheneu; 2001.

15. Taddei JA, Colugnati FA, Rodrigues EM. Transição nutricional em menores de 5 anos: evidência dos inquéritos antropométricos brasileiros. In: Cardoso AL, Lopes LA, Taddei JA, editores. Tópicos atuais em nutrição pediátrica. São Paulo: Atheneu; 2004. p. 11-43.

16. Batista Filho M, Rissin A. A transição nutricional no Brasil: tendências regionais e temporais. Cad Saude Publica 2003;9 (Suppl 1):S181-91. 
17. Biscegli TS, Corrêa CE, Romera J, Hernandez JL. Nutritional status and prevalence of iron deficiency in children enrolled in a day care center. Rev Paul Pediatr 2006;24:323-9.

18. Biscegli TS, Corrêa CE, Romera J, Candido AB. Nutritional status and iron deficiency among children enrolled in a day care center before and after 15 months of nutritional management. Rev Paul Pediatr 2008;26:124-9.

19. Biscegli TS, Polis LB, Santos LM, Vicentin M. Nutritional status and neurodevelopment of children enrolled in a day care center. Rev Paul Pediatr 2007;25:337-42.

20. Santos AL, Leão LS. Anthropometric profile of preschool children of a day care center in Duque de Caxias, Rio de Janeiro, Brazil. Rev Paul Pediatr 2008;26:218-24.

21. Machado EH, Brasil AL, Palma D, Taddei JA. Nutritional condition and prevalence of anemia in children enrolled in daycare centers. Rev Paul Pediatr 2005;23:21-6.

22. Taddei JA, Cannon MJ, Warner L, Souza P, Vitalle S, Palma D et al. Nutritional gains of underprivileged children attending a day care center in S. Paulo City, Brazil: a nine month follow-up study. Rev Bras Epidemiol 2000;3:29-37.

23. Cardoso GS, Santana AD, Aguiar CP. Prevalência e aspectos epidemiológicos da giardíase em creches no município de Aracaju, SE, Brasil. Rev Soc Bras Med Trop 1995;28:25-31.

24. Santos ME, Ogando T, Fonseca BP, Junior CE, Barçante JM [serial on the Internet]. Ocorrência de enteroparasitos em crianças atendidas no programa de saúde da família de uma área de abrangência do município de Vespasiano, Minas Gerais, Brasil. Rev Eletr Enferm [cited 2009 Jul 23]. Available from: http://www.fen.ufg.br/revista/revista8_1/pdf/v8n1a04.pdf

25. Steketee RW, Reid S, Cheng T, Stoebig JS, Harrington RG, Davis JP. Recurrent outbreaks of giardiasis in a child day care center, Wisconsin. Am J Public Health 1989;79:485-90.

26. Wharton M, Spiegel RA, Horan JM, Tauxe RV, Wells JG, Barg N et al. A large outbreak of antibiotic-resistant shigellosis at a mass gathering. J Infect Dis 1990;162:1324-8.

27. Gurgel RQ, Cardoso GS, Silva AM, Santos LM, Oliveira RC. Creche: ambiente expositor ou protetor nas infestações por parasitas intestinais em Aracaju, SE. Rev Soc Bras Med Trop 2005;38:267-9.

28. De la Ossa Merlano N, Falconar A, Llinás Solano HJ, Romero Vivas CM Manifestaciones clínicas y factores de riesgo asociados a la infección por Cryptosporidium em pacientes de Barranquilla y tres municipios del Atlántico (Colombia). Salud Uninorte 2007;23:19-31.

29. Quadros RM, Marques S, Arruda AA, Delfes PS, Medeiros IA. Parasitas intestinais em centros de educação infantil municipal de Lages, SC, Brasil. Rev Soc Bras Med Trop 2004;37:422-3.

30. Silva JO, Franceschini SA, Candido RC. Presença de leveduras em mucosas e fezes de indivíduos aparentemente saudáveis e de pessoas com sintomas de infecção fúngica. Rev Inst Adolfo Lutz 2002;61:113-20.

31. Mendes-Giannini MJ, Melhem MS. Infecções fúngicas. In: Ferreira AW, Ávila SL. Diagnóstico laboratorial das principais doenças infecciosas e auto-imunes. Rio de Janeiro: Guanabara Koogan; 1996. p. 226-50.

32. Warren NG, Hazen KC. Candida, Cryptococcus and other yeasts of medical importance In: Murray PR, Baron EJ, Pfaller MA, Tenover FC, Yolken RH, editors. Manual of clinical microbiology. $7^{\text {a }}$ ed. Washington: ASM Press; 1999. p. 1184-99.

33. Forbes D, Ee L, Camer-Pesci P, Ward P, Murphay M. Faecal candida and diarrhoea. Arch Dis Child 2001;84:328-31. 